\title{
BMJ Open Cost-effectiveness analysis of cetuximab combined with chemotherapy as a first- line treatment for patients with RAS wild-type metastatic colorectal cancer based on the TAILOR trial
}

\author{
Huijuan Wang (D) , ${ }^{1}$ Lingfei Huang, ${ }^{1}$ Peng Gao, ${ }^{1}$ Zhengyi Zhu, ${ }^{1}$ Weifeng Ye, ${ }^{1}$ \\ Haiying Ding, ${ }^{2}$ Luo Fang ${ }^{1}$
}

To cite: Wang $\mathrm{H}$, Huang $\mathrm{L}$, Gao P, et al. Cost-effectiveness analysis of cetuximab combined with chemotherapy as a firstline treatment for patients with RAS wild-type metastatic colorectal cancer based on the TAILOR trial. BMJ Open 2020;10:e030738. doi:10.1136/ bmjopen-2019-030738

- Prepublication history and additional material for this paper are available online. To view these files, please visit the journal online (http://dx.doi. org/10.1136/bmjopen-2019030738).

Received 30 March 2019 Revised 11 December 2019 Accepted 17 December 2019

Check for updates

(c) Author(s) (or their employer(s)) 2020. Re-use permitted under CC BY-NC. No commercial re-use. See rights and permissions. Published by BMJ.

${ }^{1}$ Department of Pharmacy, The Children's Hospital, Zhejiang University School of Medicine, National Clinical Research Center for Child Health, Hangzhou, China

${ }^{2}$ Department of Pharmacy,

Zhejiang Cancer Hospital, Hangzhou, China

Correspondence to

Dr Luo Fang;

fangluo@zju.edu.cn

\section{ABSTRACT}

Objectives Cetuximab plus leucovorin, fluorouracil and oxaliplatin (FOLFOX-4) is superior to FOLFOX-4 alone as a first-line treatment for patients with metastatic colorectal cancer with RAS wild-type (RAS wt mCRC), with significantly improved survival benefit by TAlLOR, an openlabel, randomised, multicentre, phase III trial. Nevertheless, the cost-effectiveness of these two regimens remains uncertain. The following study aims to determine whether cetuximab combined with FOLFOX-4 is a cost-effective regimen for patients with specific RAS wt $\mathrm{mCRC}$ in China. Design A cost-effectiveness model combined decision tree and Markov model was built to simulate pateints with RAS wt mCRC based on health states of dead, progressive and stable. The health outcomes from the TAILOR trial and utilities from published data were used respectively. Costs were calculated with reference to the Chinese societal perspective. The robustness of the results was evaluated by univariate and probabilistic sensitivity analyses.

Participants The included patients were newly diagnosed Chinese patients with fully RAS wt mCRC.

Interventions First-line treatment with either cetuximab plus FOLFOX-4 or FOLFOX-4.

Main outcome measures The primary outcomes are costs, quality-adjusted life-years (QALYs) and incremental cost-effectiveness ratios (ICERs).

Results Baseline analysis disclosed that the QALYs was increased by 0.383 caused by additional cetuximab, while an increase of US\$62947 was observed in relation to FOLFOX-4 chemotherapy. The ICER was US\$164044 per QALY, which exceeded the willingness-to-pay threshold of US\$28106 per QALY.

Conclusions Despite the survival benefit, cetuximab combined with FOLFOX-4 is not a cost-effective treatment for the first-line regime of patients with RAS wt mCRC in China.

Trial registration number TAILOR trial (NCT01228734); Post-results.

\section{INTRODUCTION}

Colorectal cancer (CRC) is the fourth most prevalent cancer worldwide. ${ }^{1}$ The agestandardised incidence and mortality of CRC
Strengths and limitations of this study

- Our study focused on metastatic colorectal cancer patients with specific RAS wild-type and first evaluated the health and economic outcomes of cetuximab combined with FOLFOX-4 as a first-line treatment based on TAILOR trial in China.

- One limitation of this study is that health states and costs due to other reasons instead of disease progression were not included in the model.

- The utilities used in the current model are based on previously published research, and an updated health utility data of the patients with metastatic colorectal cancer in Chinese populations might improve accuracy and robustness of the reported findings.

is 20.7/100 000 and 9.6/100 000 of Chinese men, and 14.4/100 000 and 6.3/100 000 of Chinese women. ${ }^{2}$ Early-stage CRC presents asymptomatically; approximately $25 \%$ of patients present with metastases at initial diagnosis, and almost $50 \%$ of people with CRC will develop metastases. ${ }^{3}$ In China, 5-year age-standardised net survival of CRC was still only $57 \%,{ }^{4}$ which is lower than that in developed countries, and long-term prognosis of CRC is poor. ${ }^{5}$ For many years, fluorouracilbased regimens, including the FOLFOX (fluorouracil, oxaliplatin and leucovorin) and FOLFIRI (fluorouracil, irinotecan and leucovorin) regimens, have been the basic treatment for metastatic CRC (mCRC) ${ }^{6}$ Over the last decade, targeted therapies such as cetuximab have been developed, and cetuximab combined with chemotherapy has been used as a first-line treatment of mCRC. ${ }^{78}$

Cetuximab is a recombinant chimeric monoclonal $\operatorname{IgG}_{1}$ antibody of epidermal growth factor receptor (EGFR) to inhibit the growth, invasion and metastasis of cancer 


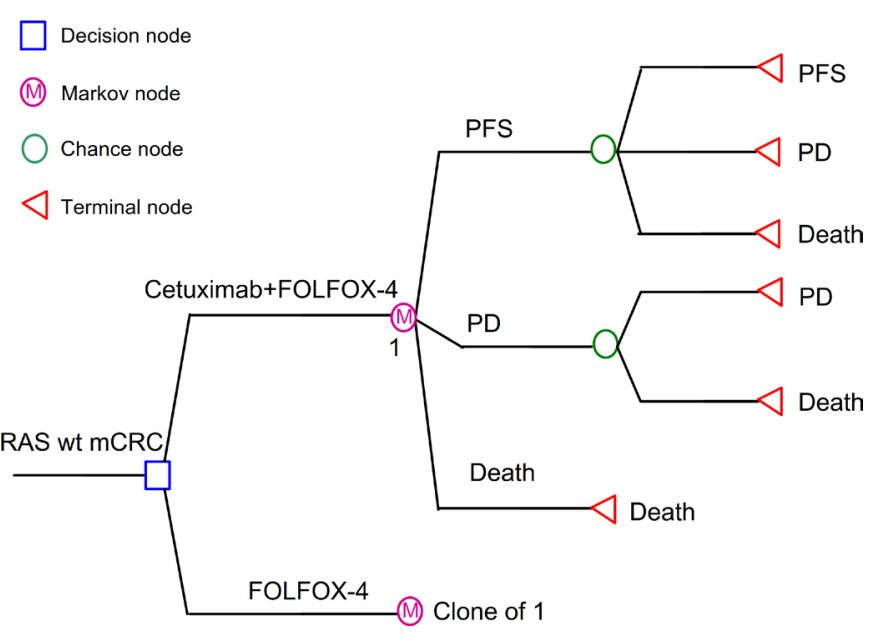

Figure 1 The decision tree and Markov model used to simulate a hypothetical cohort of patients with RAS wt $\mathrm{mCRC}$ based on the TAILOR trial. Two groups were analysed: group 1, patients with metastatic colorectal cancer treated with cetuximab + FOLFOX-4; and group 2, patients with metastatic colorectal cancer treated with FOLFOX-4. A Markov model comprised three health states (PFS, PD and death). FOLFOX, oxaliplatin, fluorouracil and leucovorin chemotherapy; mCRC, metastatic colorectal cancer; PD, progressive disease; PFS, progression-free survival; wt, wildtype.

cells. ${ }^{9}$ Cetuximab has been indicated to be effective for treatment of patients with extended RAS (KRAS/NRAS, exons 2-4) wild-type (wt) CRCs and was recommended by colorectal cancer guidelines of the Chinese Society of Clinical Oncology (CSCO). ${ }^{10-15}$ The combination of cetuximab with oxaliplatin-based chemotherapy has been shown to improve the clinical outcomes of first-line treatment in patients with RAS wt mCRC by phase II clinical study. ${ }^{11}{ }^{14}$ These findings were finally confirmed in the TAILOR trial (a brief overview of the TAILOR trial is presented in online supplementary appendix 1), the first randomised phase III trial to evaluate the efficacy and tolerability of cetuximab plus FOLFOX-4 in Chinese patients with RAS wt mCRC. ${ }^{16}$

The TAILOR trial was the first prospective, parallelgroup, multicentre, randomised, phase III trial to evaluate the efficacy and safety of first-line therapy with cetuximab plus FOLFOX regimen in patients with RAS wt mCRC. The combination of cetuximab with the FOLFOX-4 regimen significantly improved the survival benefit of patients with mCRC with wt RAS. The trial provided robust evidence to support the FOLFOX plus cetuximab regimen as a first-line treatment for patients with RAS wt mCRC. ${ }^{16}$ However, adding cetuximab to FOLFOX-4 is also accompanied by related adverse reactions and high costs. The cost-effectiveness of the combined strategy was unclear. Therefore, in the present study, we conducted a cost-effectiveness analysis to evaluate cetuximab plus FOLFOX-4 versus FOLFOX-4 alone in the first-line treatment setting for patients with mCRC based on the TAILOR trial with respect to the Chinese societal perspective by using a decision tree plus Markov model.
MATERIALS AND METHODS

\section{Patients and treatments}

TAILOR was the first prospectively phase III study to evaluate the clinical benefit of additional cetuximab and first-line FOLFOX-4 treatment in the RAS wt population. The included patients were 393 newly diagnosed Chinese patients with fully RAS wt mCRC ( $50.4 \%$ of colon, $49.1 \%$ of rectum and $0.5 \%$ of both) who met the eligibility criteria for the phase III clinical trial (TAILOR). The average age of enrolled patients was 56 years old (range: 21-83); male patients accounted for $66.7 \% .{ }^{16}$ All patients were randomly assigned (1:1) to receive either cetuximab plus FOLFOX-4 or FOLFOX-4 alone according to unstratified block randomisation.

Based on the TAILOR trial, the 14-day treatment scheme was as follows: FOLFOX-4 included an oxaliplatin $85 \mathrm{mg} / \mathrm{m}^{2}$ infusion, calcium leucovorin $200 \mathrm{mg} / \mathrm{m}^{2}$ infusion and 5-fluorouracil as a bolus of $400 \mathrm{mg} / \mathrm{m}^{2} /$ day intravenously followed by $600 \mathrm{mg} / \mathrm{m}^{2} /$ day infusion on day 1 and day 2. Every patient received FOLFOX-4 according to the protocol. For the cetuximab plus FOLFOX-4 group, cetuximab was administered at a dose of $250 \mathrm{mg} / \mathrm{m}^{2}$ every 7 days. Treatment continued until disease progression or until additional reasons caused discontinuation. Patients with progression were assumed to receive FOLFIRI second-line chemotherapy as post-progression strategy based on the recommendations of Chinese guidelines. ${ }^{15}$

\section{Model structure}

A dynamic Markov model was constructed by using TreeAge pro Suite (TreeAge Software, Williamstown, Massachusetts, USA) to assess the cost-effectiveness of adding cetuximab for the treatment of RAS wt mCRC. The model included three health states: progression-free survival (PFS), progressive disease (PD) and death. All patients were assumed to be PFS state at the time of enrolment and could remain in the original state or move to the other states at the end of each cycle depending on the transition probability (figure 1 ). The model cycle length was 1 month. The time horizon was 10 years, because the median overall survival (OS) time of patients with mCRC was $<2$ years and the probability of survival in 10 years was zero in the TAILOR trial. ${ }^{16}$ Both the costs and effectiveness were discounted at $3 \%$ annually. The main model outputs included the total costs and the quality-adjusted life-years (QALYs).

\section{Transition probabilities}

The transition probabilities to disease progression and death for cetuximab plus FOLFOX-4 and FOLFOX-4 alone were based on the parametric survival curves of PFS and OS from the publication of the TAILOR clinical trial. In the patients with RAS wt mCRC, additional cetuximab significantly improved the median PFS of FOLFOX-4 (9.2 vs 7.4 months; HR, $0.69 ; 95 \% \mathrm{CI}, 0.54$ to 0.89 ) and $\mathrm{OS}$ (20.7 vs 17.8 months; HR, 0.76 ; $95 \% \mathrm{CI}, 0.61$ to 0.96$){ }^{16}$ The Weibull survival function $S(t)=\exp \left(-\lambda t^{\prime}\right)$ was employed to fit the Kaplan-Meier PFS and OS probabilities. The 
Table 1 Parameters inputs

\begin{tabular}{|c|c|c|}
\hline Parameters & Values (ranges) & Description and reference \\
\hline \multicolumn{3}{|l|}{ Clinical data } \\
\hline Weibull parameters PFS of cetuximab +FOLFOX-4 & $\begin{array}{l}\text { Scale }=0.0445(0.036-0.059) \\
\text { Shape }=1.2248 ; R^{2}=0.996218\end{array}$ & 16 \\
\hline Weibull parameters PFS of FOLFOX-4 & $\begin{array}{l}\text { Scale }=0.0462(0.036-0.062) \\
\text { Shape }=1.4075 ; R^{2}=0.992504\end{array}$ & 16 \\
\hline Weibull parameters OS of cetuximab +FOLFOX-4 & $\begin{array}{l}\text { Scale }=0.0614(0.053-0.073) \\
\text { Shape }=0.8404 ; R^{2}=0.992250\end{array}$ & 16 \\
\hline Weibull parameters OS of FOLFOX-4 & $\begin{array}{l}\text { Scale=0.0417 }(0.035-0.052) \\
\text { Shape }=1.0205 ; R^{2}=0.991371\end{array}$ & 16 \\
\hline \multicolumn{3}{|l|}{ Costs (US\$) } \\
\hline Cost of cetuximab per $100 \mathrm{mg}$ & $187.7(150.2-225.2)$ & Local charge* $^{*}$ \\
\hline Cost of oxaliplatin per $50 \mathrm{mg}$ & $338.4(270.7-406.1)$ & Local charge $^{*}$ \\
\hline Fluorouracil per $250 \mathrm{mg}$ & $0.9(0.8-1.1)$ & Local charge $^{*}$ \\
\hline Leucovorin per $100 \mathrm{mg}$ & $4.6(3.7-5.6)$ & Local charge* $^{*}$ \\
\hline Hospitalisation per day & $4.4(3.5-5.2)$ & Local charge* $^{*}$ \\
\hline Disease monitor per treatment scheme & $150(120-180)$ & Local charge* $^{*}$ \\
\hline Time costs per day & $29.5(23.6-35.4)$ & Local charge $†$ \\
\hline Costs of FOLFOX-4 per cycle & 5286.7 (4229.4-6344.0) & Calculation* \\
\hline Costs of salvage therapy per cycle & $2388.3(1910.4-2865.6)$ & Calculation* \\
\hline \multicolumn{3}{|l|}{ Cost data of managing AEs } \\
\hline Costs of neutropaenia per event & $197.8(158.3-237.4)$ & Local charge* $^{*}$ \\
\hline Costs of thrombocytopenia per event & $1022.6(818.1-1227.1)$ & Local charge $^{*}$ \\
\hline Costs of skin reactions per event & $1.8(1.4-2.1)$ & Local charge* $^{*}$ \\
\hline \multicolumn{3}{|l|}{ Utilities } \\
\hline Utility of PFS & $0.85(0.68-1.00)$ & 19 to 20 \\
\hline Utility of PD & $0.78(0.62-0.94)$ & 21 \\
\hline
\end{tabular}

*Authorised fee by the Health Commission of Zhejiang Province.

†The average daily salary released by Chinese National Bureau of Statistics in 2018.

AEs, adverse events; FOLFOX, oxaliplatin, fluorouracil and leucovorin chemotherapy; OS, overall survival; PD, progressive disease; PFS, progression-free survival.

estimated scale $(\lambda)$, shape parameters $(\gamma)$ and adjusted $\mathrm{R}^{2}$ are presented in table 1 . The calibration curve showed in online supplementary appendix 2 . The shape parameter $(\gamma>0)$ allows increasing, constant or decreasing hazards, when $\gamma>1$ the risk increases over time, $0<\gamma<1$ the risk decreases over time. The duration of the PFS and PD states was calculated using the area under the PFS and OS survival curves. The transition probability from PFS to $\mathrm{PD}$ in each cycle was calculated based on the formulation: $1-\exp \left(\lambda[\mathrm{t}-1]^{\gamma}-\lambda \mathrm{t}^{\gamma}\right)$, where $t$ is the current stage in the Markov model. ${ }^{17}$ The difference between the OS and PFS estimated from the models was used to calculate the transition probability from PD to death. ${ }^{18}$

\section{Costs and utilities}

Costs were calculated with respect to the Chinese societal perspective. Costs of both first-line and second-line treatments were included. Direct costs as well as indirect costs were all considered. RAS mutation screen was assumed as a routine test of all patients because it was recommended as molecular diagnosis for all patients with $\mathrm{mCRC}$ regardless of the regimen used. Direct costs incorporated the costs of drugs, hospitalisations and tests for oncology, and treatments for grade $3 / 4$ adverse events (AEs) were referred to the standards fee authorised by the Health Commission of Zhejiang Province. Medication costs were calculated based on a case patient with a height of $161 \mathrm{~cm}$, a weight of $62 \mathrm{~kg}$ and a body surface area of $1.66 \mathrm{~m}^{2}$. Moreover, the grade 3/4 AEs data were derived from the TAILOR trial. And the related costs were estimated according to the incidence rate (online supplementary appendix 1) and responding unit cost of each AE. The management of AEs was based on published guidelines. Neutropaenia and thrombocytopenia were managed with recombinant human granulocyte colony stimulating factor and recombinant human thrombopoietin, respectively, for 7 days at adult dose; skin reactions were 
managed with $0.1 \%$ momethasone furoate cream; fatigue had no specific medical management. Indirect costs included time costs and were estimated according to the average salary (US $\$ 29.5$ per day) released by the Chinese National Bureau of Statistics in 2018 with 6 and 8 days of hospital stays for FOFLFOX-4 alone and cetuximab plus FOLFOX-4, respectively. An exchange rate of Chinese Yuan Renminbi to US dollar (6.9:1, 19 November 2018) were used. The estimated costs are presented in table 1 .

The effectiveness data were calculated based on the health-related quality of life. Health state utility scores were referenced from previously published studies. We assigned a utility of 0.85 for the PFS state, ${ }^{19} 0.78$ for the PD state in subsequent second-line chemotherapy and 0 for death. ${ }^{20}{ }^{21}$ The health state utility scores are listed in table 1 .

\section{Cost-effectiveness analysis}

The incremental costs per QALY were used to evaluate the economics of both regimens. An intervention was labelled as dominant when it was less expensive and more effective than a contrasted intervention. If the ICER was less than the willingness-to-pay (WTP) threshold of US\$28 106/QALY, which was set as 3× per capita GDP of China in 2018 on the basis of the WHO threshold recommendation for cost-effectiveness analyses, more effective and more expensive intervention was considered costeffective. $^{22} 23$

\section{Sensitivity analysis}

The robustness and uncertainty of model parameters were estimated by univariate and probabilistic sensitivity analysis (PSA), respectively. Univariate sensitivity analysis was performed by varying parameters by $\pm 20 \%,{ }^{23}$ in addition to the discount rate ranging from $0 \%$ to $8 \%$, and all other parameters remain unchanged. The data range was displayed in table 1 . A tornado diagram was used to evaluate the robustness of the results, which was used to reflect the underlying effect of the parameters on the outputs. Furthermore, PSA was performed with 1000 Monte Carlo simulations. All parameters were randomly sampled from the distributions of parameter (beta distribution for utilities and gamma distribution for costs) at the same time. ${ }^{2425}$

\section{Patient and public involvement}

The study design was a secondary data analysis and did not directly involve patients or the public.

\section{RESULTS}

\section{Base case analysis}

Base case analysis displayed that the total cost of cetuximab plus group was US $\$ 137606$ compared with US $\$ 74659$ for FOLFOX-4 alone group. For effectiveness, the combined group yielded an incremental 0.383 QALYs compared with chemotherapy alone (1.842 vs 1.459 QALYs). The ICER was US $\$ 164044$ per QALY, which was significantly
Table 2 Results of base-case analysis

\begin{tabular}{|c|c|c|}
\hline & $\begin{array}{l}\text { Cetuximab + } \\
\text { FOLFOX-4 }\end{array}$ & FOLFOX-4 \\
\hline \multicolumn{3}{|l|}{ Costs(US\$) } \\
\hline PFS state & 99553 & 41482 \\
\hline PD state & 38053 & 33178 \\
\hline Total & 137606 & 74659 \\
\hline Incremental costs & - & 62947 \\
\hline \multicolumn{3}{|l|}{ Effectiveness (QALYs) } \\
\hline PFS state & 0.81 & 0.56 \\
\hline PD state & 1.04 & 0.90 \\
\hline Total & 1.84 & 1.46 \\
\hline Incremental effectiveness & - & 0.383 \\
\hline $\begin{array}{l}\text { Incremental cost/ } \\
\text { effectiveness (US\$) }\end{array}$ & & 164044 \\
\hline
\end{tabular}

Results are based on probabilistic analysis and are discounted at $3 \%$ per annum rate.

FOLFOX, oxaliplatin, fluorouracil and leucovorin; PD, progressive disease; PFS, progression-free survival; QALY, quality-adjusted life-year.

higher than the WTP threshold of US\$28106. Detailed information regarding base-case analysis is shown in table 2.

\section{Sensitivity analysis}

The results of univariate sensitivity analyses are depicted in the tornado diagram in figure 2 . The parameters that had the greatest impact on the ICERs were the median OS and PFS time in the cetuximab plus FOLFOX-4 group. When the OS time in the combined regimen varied from 16.6 to 24.8 months, the ICER decreased from US $\$ 1029565$ to US $\$ 111088$ per QALY. The other parameters, costs of the PFS state for the two groups, utility of the PFS state, discount rate, and costs of cetuximab were all important influential factors of ICER. For all of the parameter variations, the ICER for the combined group always exceeded the WTP per QALY for China.

The cost-effectiveness acceptability curves (figure 3A) showed the probability that the cetuximab plus FOLFOX-4 group was cost-effective across increasing WTP values. The results of the PSA showed cetuximab was not cost-effective unless the WTP value was > US $\$ 40000$ per QALY. In addition, the scatterplot depicted incremental cost vs incremental QALY for Monte Carlo simulations, with an oblique line showing the WTP threshold of US\$28 106 per QALY (figure 3B). Approximately 20\% of the simulations fell in the fourth quadrant, suggesting that additional cetuximab treatment was more costly but less effective than chemotherapy. A total of $80 \%$ of the simulations fell in the first quadrant, which showed that additional cetuximab treatment was more costly and more effective than chemotherapy. All the points were above the WTP threshold line, indicating that cetuximab 
Tornado Analysis (ICER)

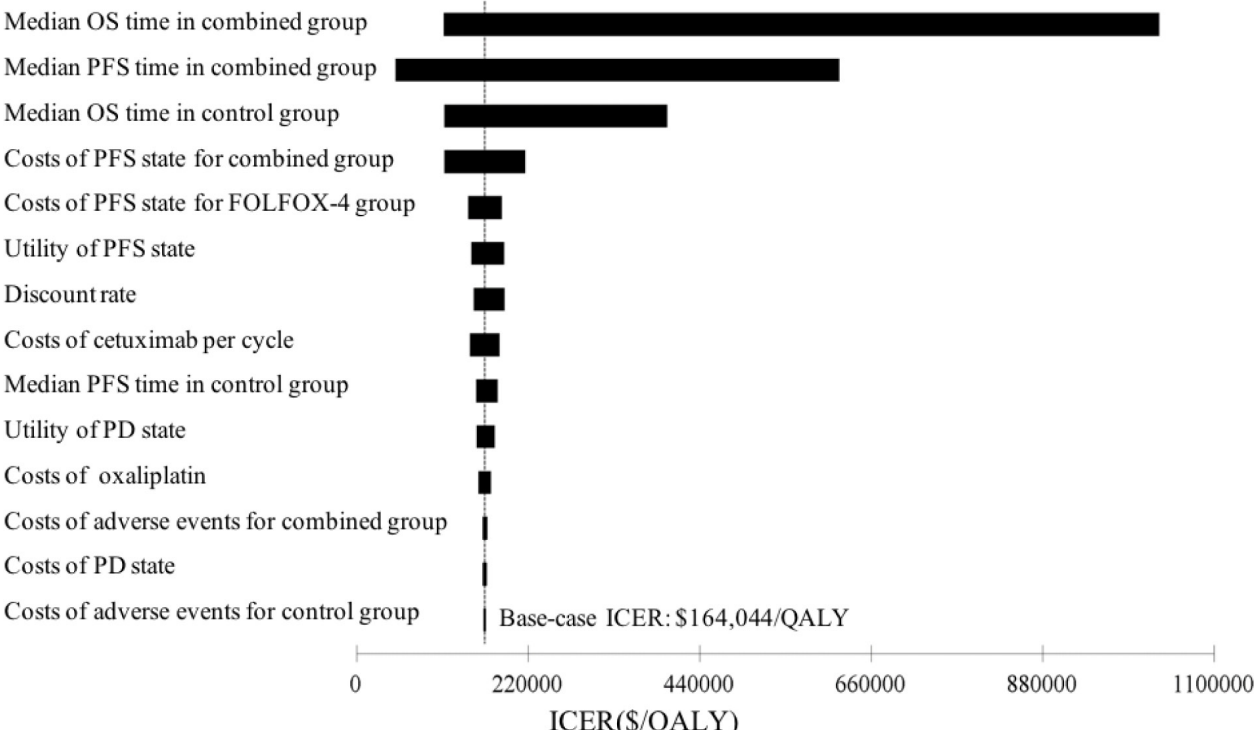

Figure 2 Tornado diagrams of one-way sensitivity analyses. Tornado diagrams show the influence of factors on the Markov model of the two strategies in the treatment of metastatic colorectal cancer. The factors are listed in descending order of their influence on ICER with variation in the factor values. ICER, incremental cost-effectiveness ratio; OS, overall survival; PD, progressive disease; PFS, progression-free survival.

plus FOLFOX-4 treatment was not cost-effective for RAS wt mCRC in China.

\section{DISCUSSION}

To our knowledge, the TAILOR trial is the first phase III study that evaluated the clinical benefit of cetuximabcombined FOLFOX regime in the first line treatment of patients with RAS wt mCRC. The trial showed a prolonged PFS of 1.8 months and OS of 2.9 months in patients with RAS wt mCRC accompanied by significantly increased costs. In the current study, we evaluate the health and economic outcomes of cetuximab as a firstline treatment for patients with RAS wt mCRC in a typical area with limited medical health resources. Because most of the high-quality evidence were conducted in western countries, many cost-effectiveness analyses for Chinese patients were based on the efficacy and safety data obtained from western countries. Therefore, the present analysis provided pharmacoeconomics evidence for the cetuximab plus FOLFOX as a first-line treatment option for patients with RAS wt mCRC in China.

According to our results, the cetuximab group provided an incremental 0.383 QALYs at an incremental cost of US\$62947 compared with control group, resulting in an ICER of US $\$ 164044$ per QALY (approximately sixfolds of WTP). One-way sensitivity analysis showed that the median OS and PFS time in the cetuximab group was the most influential variable parameter. Other independent influence parameters included the costs of the PFS state, where the price of cetuximab was the most important driver. Our results corroborate the findings of many previous works. An economic analysis performed in the USA indicated that RAS mutation screening and cetuximab for patients with RAS wt mCRC produced an ICER of about US $\$ 650000$ per year of life in comparison with no anti-EGFR therapy. ${ }^{26}$ Furthermore, a prospective cost-effectiveness analysis for patients with KRAS wt tumours in Canada showed that the incremental costeffectiveness ratio is US $\$ 120061$ per life-year gained and the incremental cost-utility ratio is US $\$ 186761$ per QALY gained. In addition, cetuximab cost and patient survival were very important variables of cost-effectiveness. ${ }^{27}$ The cost-effectiveness analysis focused on cetuximab in patients with mCRC was systematically reviewed by Huxley et $a l,{ }^{28}$ but the economic analysis with respect to this problem was still inadequate in China. Wu et al used data from Chinese medical insurance perspective and revealed that the ICER of US\$27 145/QALY for the cetuximab plus FOLFIRI treatment regimen was close to the WTP threshold in patients with KRAS wt mCRC, but the survival data were derived from two trials, the CRYSTAL trial and the FIRE-3 study, in western regions. ${ }^{29}$ However, the TAILOR trial was conducted in China, so the present study may provide more solid pharmacoeconomic evidence for Chinese patients. Various contributors, such as populations with different genetic mutation statuses, patients treated with different chemotherapy regimens and different clinical practices between western and east regions, may cause different findings.

Our study has some limitations. First, the modelling that was performed to extrapolate clinical survival rather than obtaining prospective observational data is an unavoidable limitation in this study. The health states and costs that were involved as a result of other causes, such as adverse events caused discontinuation, were not included in this model. Second, the TAILOR study did not report 


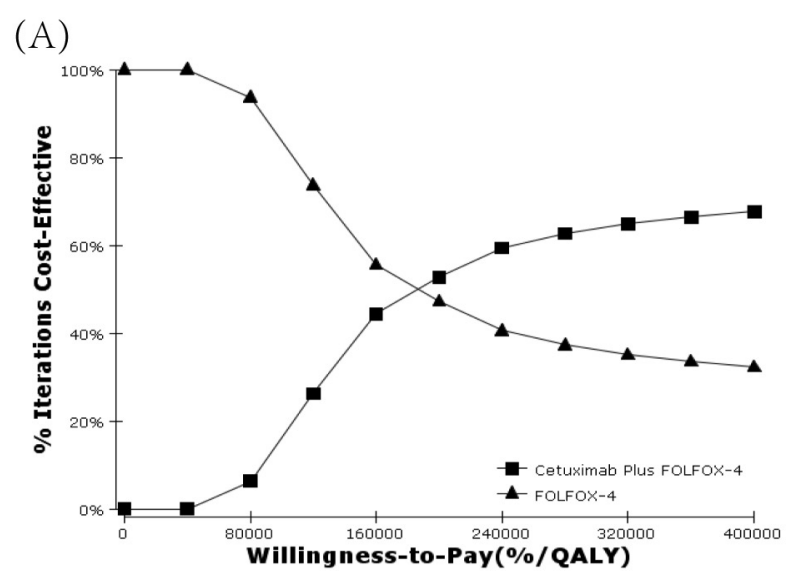

(B)

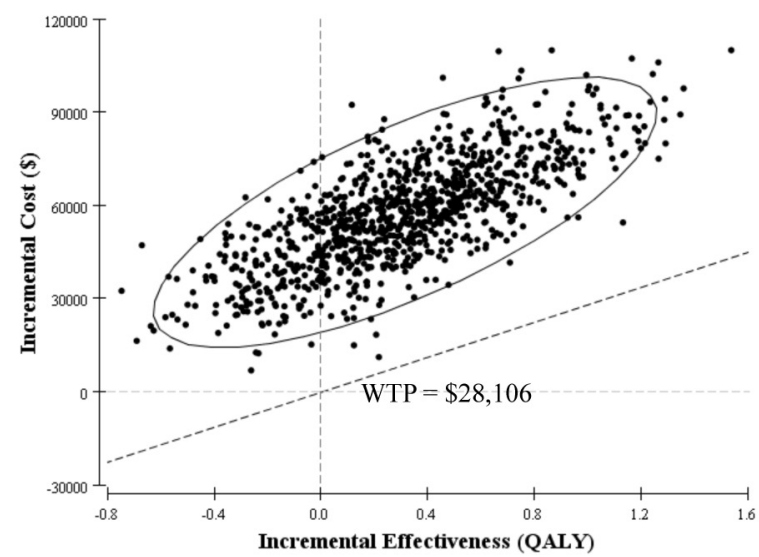

Figure 3 Probabilistic sensitivity analysis. (A) Costeffectiveness acceptability curve. The cost-effectiveness acceptability frontier shows the probability of strategies being cost-effective in two strategies. (B) Scatterplot of 1000 iterations of Monte Carlo simulations. FOLFOX, oxaliplatin, fluorouracil and leucovorin; QALY, quality-adjusted life-year; WTP, willingness-to-pay

the data on quality of life, so utilities for the states of PFS and PD were derived from published data. An updated health utility data for patients with mCRC in Chinese populations might improve the accuracy and robustness of the reported findings. Third, the duration of hospitalisation and periodicity of tests were estimated by experts. The sensitivity analysis indicated that the factors had little impact on the results. Finally, the model did not fully evaluate subgroup situation, such as liver metastases patients, and second- or best supportive treatment combined with other regimens.

Despite these limitations, the results of our analysis are still reasonable. Our study, which was based on clinical research in the Chinese population, indicated that adding cetuximab to FOLOX is unlikely to be a costeffective strategy for patients with RAS wt mCRC in China. The reported conclusions may be helpful to physicians, patients and health management agency in their decision-making processes.

Contributors HW, LH and LF designed the study and drafted the manuscript. $H W, P G$ and WY collected the data, performed the literature search. HW and HD constructed the decision tree plus Markov model. LF, HW and ZZ interpreted the data and revised the manuscript. All authors approved the manuscript.

Funding This work was supported by Zhejiang Provincial Program for 151 Talents (Luo Fang).

Competing interests None declared.

Patient consent for publication Not required.

Ethics approval All procedures performed in studies involving human participants were in accordance with the ethical standards of medical ethics committee of The Children's Hospital, Zhejiang University School of Medicine. Additionally, the study was performed in accordance with the ethical standards put forth in the 1964 Declaration of Helsinki.

Provenance and peer review Not commissioned; externally peer reviewed.

Data availability statement Data are available in a public, open access repository. Extra data can be accessed via the Dryad data repository at http://datadryad.org/ with the doi: 10.5061/dryad.15dv41ntb.

Open access This is an open access article distributed in accordance with the Creative Commons Attribution Non Commercial (CC BY-NC 4.0) license, which permits others to distribute, remix, adapt, build upon this work non-commercially, and license their derivative works on different terms, provided the original work is properly cited, appropriate credit is given, any changes made indicated, and the use is non-commercial. See: http://creativecommons.org/licenses/by-nc/4.0/.

\section{ORCID iD}

Huijuan Wang http://orcid.org/0000-0001-9636-4615

\section{REFERENCES}

1 Bray F, Ferlay J, Soerjomataram I, et al. Global cancer statistics 2018: GLOBOCAN estimates of incidence and mortality worldwide for 36 cancers in 185 countries. CA Cancer J Clin 2018;68:394-424.

2 Chen W, Sun K, Zheng R, et al. Cancer incidence and mortality in China, 2014. Chin J Cancer Res 2018;30:1-12.

3 Van Cutsem E, Cervantes A, Nordlinger B, et al. Metastatic colorectal cancer: ESMO clinical practice guidelines for diagnosis, treatment and follow-up. Ann Oncol 2014;25 Suppl 3:iii1-9.

4 Allemani C, Matsuda T, Di Carlo V, et al. Global surveillance of trends in cancer survival 2000-14 (CONCORD-3): analysis of individual records for 37513025 patients diagnosed with one of 18 cancers from 322 population-based registries in 71 countries. The Lancet 2018;391:1023-75.

5 DeSantis CE, Lin CC, Mariotto AB, et al. Cancer treatment and survivorship statistics, 2014. CA Cancer J Clin 2014;64:252-71.

6 Douillard JY, Cunningham D, Roth AD, et al. Irinotecan combined with fluorouracil compared with fluorouracil alone as first-line treatment for metastatic colorectal cancer: a multicentre randomised trial. The Lancet 2000;355:1041-7.

7 Van Cutsem E, Köhne C-H, Hitre E, et al. Cetuximab and chemotherapy as initial treatment for metastatic colorectal cancer. $N$ Engl J Med 2009;360:1408-17.

8 Saltz LB, Clarke S, Díaz-Rubio E, et al. Bevacizumab in combination with oxaliplatin-based chemotherapy as first-line therapy in metastatic colorectal cancer: a randomized phase III study. JCO 2008;26:2013-9.

9 Ciardiello F, Tortora G. Egfr antagonists in cancer treatment. N Engl J Med 2008;358:1160-74

10 Bokemeyer C, Bondarenko I, Makhson A, et al. Fluorouracil, leucovorin, and oxaliplatin with and without cetuximab in the first-line treatment of metastatic colorectal cancer. JCO 2009;27:663-71.

11 Bokemeyer C, Bondarenko I, Hartmann JT, et al. Efficacy according to biomarker status of cetuximab plus FOLFOX-4 as first-line treatment for metastatic colorectal cancer: the OPUS study. Ann Oncol 2011;22:1535-46.

12 Van Cutsem E, Köhne C-H, Láng I, et al. Cetuximab Plus Irinotecan, Fluorouracil, and Leucovorin As First-Line Treatment for Metastatic Colorectal Cancer: Updated Analysis of Overall Survival According to Tumor KRAS and BRAF Mutation Status. JCO 2011;29:2011-9.

13 Van Cutsem E, Lenz H-J, Köhne C-H, et al. Fluorouracil, Leucovorin, and Irinotecan Plus Cetuximab Treatment and RAS Mutations in Colorectal Cancer. JCO 2015;33:692-700.

14 Bokemeyer $\mathrm{C}$, Köhne C-H, Ciardiello F, et al. Folfox4 plus cetuximab treatment and Ras mutations in colorectal cancer. Eur $J$ Cancer 2015;51:1243-52.

15 Diagnosis And Treatment Guidelines For Colorectal Cancer Working Group CSOCOC, colorectal cancer working group C. Chinese 
Society of clinical oncology (CSCO) diagnosis and treatment guidelines for colorectal cancer 2018 (English version). Chin J Cancer Res 2019;31:117-34.

16 Qin S, Li J, Wang L, et al. Efficacy and tolerability of first-line cetuximab plus leucovorin, fluorouracil, and oxaliplatin (folfox-4) versus folfox-4 in patients with ras wild-type metastatic colorectal cancer: the open-label, randomized, phase iii tailor trial. JCO 2018;36:3031-9.

17 Diaby V, Adunlin G, Montero AJ. Survival modeling for the estimation of transition probabilities in model-based economic evaluations in the absence of individual patient data: a tutorial. Pharmacoeconomics 2014;32:101-8.

18 Chouaid C, Luciani L, LeLay K, et al. Cost-Effectiveness analysis of afatinib versus gefitinib for first-line treatment of advanced EGFRmutated advanced non-small cell lung cancers. J Thorac Oncol 2017; 12:1496-502.

19 Ramsey SD, Andersen MR, Etzioni R, et al. Quality of life in survivors of colorectal carcinoma. Cancer 2000;88:1294-303.

20 Graham CN, Hechmati G, Hjelmgren J, et al. Cost-Effectiveness analysis of panitumumab plus mFOLFOX6 compared with bevacizumab plus mFOLFOX6 for first-line treatment of patients with wild-type Ras metastatic colorectal cancer. Eur J Cancer 2014;50:2791-801.

21 Riesco-Martínez MC, Berry SR, Ko Y-J, et al. Cost-Effectiveness Analysis of Different Sequences of the Use of Epidermal Growth Factor Receptor Inhibitors for Wild-Type KRAS Unresectable Metastatic Colorectal Cancer. J Oncol Pract 2016;12:e710-23.
22 Murray CJL, Evans DB, Acharya A, et al. Development of who guidelines on generalized cost-effectiveness analysis. Health Econ 2000;9:235-51.

23 Elbasha EH, Messonnier ML. Cost-Effectiveness analysis and health care resource allocation: decision rules under variable returns to scale. Health Econ 2004;13:21-35.

24 Briggs $\mathrm{AH}$. Handling uncertainty in cost-effectiveness models. Pharmacoeconomics 2000;17:479-500.

25 Briggs $\mathrm{AH}$, Weinstein MC, Fenwick EAL, et al. Model parameter estimation and uncertainty analysis: a report of the ISPOR-SMDM modeling good research practices Task force working Group-6. Med Decis Making 2012;32:722-32.

26 Behl AS, Goddard KAB, Flottemesch TJ, et al. Cost-Effectiveness analysis of screening for KRAS and BRAF mutations in metastatic colorectal cancer. J Nat/ Cancer Inst 2012;104:1785-95.

27 Mittmann N, Au H-J, Tu D, et al. Prospective cost-effectiveness analysis of cetuximab in metastatic colorectal cancer: evaluation of national cancer Institute of Canada clinical Trials Group CO.17 trial. J Natl Cancer Inst 2009;101:1182-92.

28 Huxley N, Crathorne L, Varley-Campbell J, et al. The clinical effectiveness and cost-effectiveness of cetuximab (review of technology appraisal No. 176) and panitumumab (partial review of technology appraisal No. 240) for previously untreated metastatic colorectal cancer: a systematic review and economic evaluation. Health Technol Assess 2017;21:1-294.

29 Wu B, Yao Y, Zhang K, et al. Ras testing and cetuximab treatment for metastatic colorectal cancer: a cost-effectiveness analysis in a setting with limited health resources. Oncotarget 2017;8:71164-72. 